\title{
Das Blumenparterre im Palmengarten
}

\author{
Hilke Steinecke
}

\section{Eindruck}

Wer den Palmengarten von der Bockenheimer Landstraße kommend über den Eingang Palmengarten betritt, stößt gleich auf eines der besonders markanten und historischen Bereiche des Palmengartens. Vor der weißen Fassade des frisch sanierten Gesellschaftshauses erstreckt sich das Blumenparterre, in dessen Mitte sich ein Wasserbecken mit Fontäne befindet, und das seitlich von zwei Alleen regelmäßig gestutzter Holländischer Linden (Tilia x vulgaris) begrenzt wird. Mit Beendigung der Renovierungsarbeiten und Wiedereröffnung des Gesellschaftshauses wurde das Blumenparterre zur Palmengartenstraße hin geöffnet und ist frei zugänglich, der Eintritt in den Palmengarten ist erst dahinter zu entrichten.

Der Rand des Blumenparterres wird jedes Jahr neu bepflanzt, wobei Farb- und Artenkombinationen des Frühlings- und Sommerflors in jeder Saison wechseln. Passend zur
Randbepflanzung ist auch die kleine Böschung vor dem Gesellschaftshaus gestaltet.

Zur Gründerzeit wurde das Blumenparterre viel opulenter bepflanzt. August Siebert beschreibt die Eingangssituation des Palmengartens in seinem Gartenführer von 1895: „Wir passieren nun eine Rhododendron-Gruppe und links ein halbrundes Blumenparterre, in dessen Mitte sich ein Springbrunnen, umgeben von einem achteckigen Stern, befindet, der mit der ausserordentlich wirkungsvollen, erst 1892 eingeführten Begonia semperflorens 'Vernon' bepflanzt ist. Die übrige Bepflanzung ist in einfacher Rabattenform und stets der Jahreszeit entsprechend gehalten. Uns rechtswendend, gelangen wir zunächst zu den an einer sanft ansteigenden Böschung liegenden eigentlichen Teppichbeeten. Es sind deren drei, die in jedem Jahre neue Muster- und Farbenzusammenstellungen niedrig bleibender Gewächse aufweisen. Das mittlere Beet zeigte im Jahre 1893 symbo-

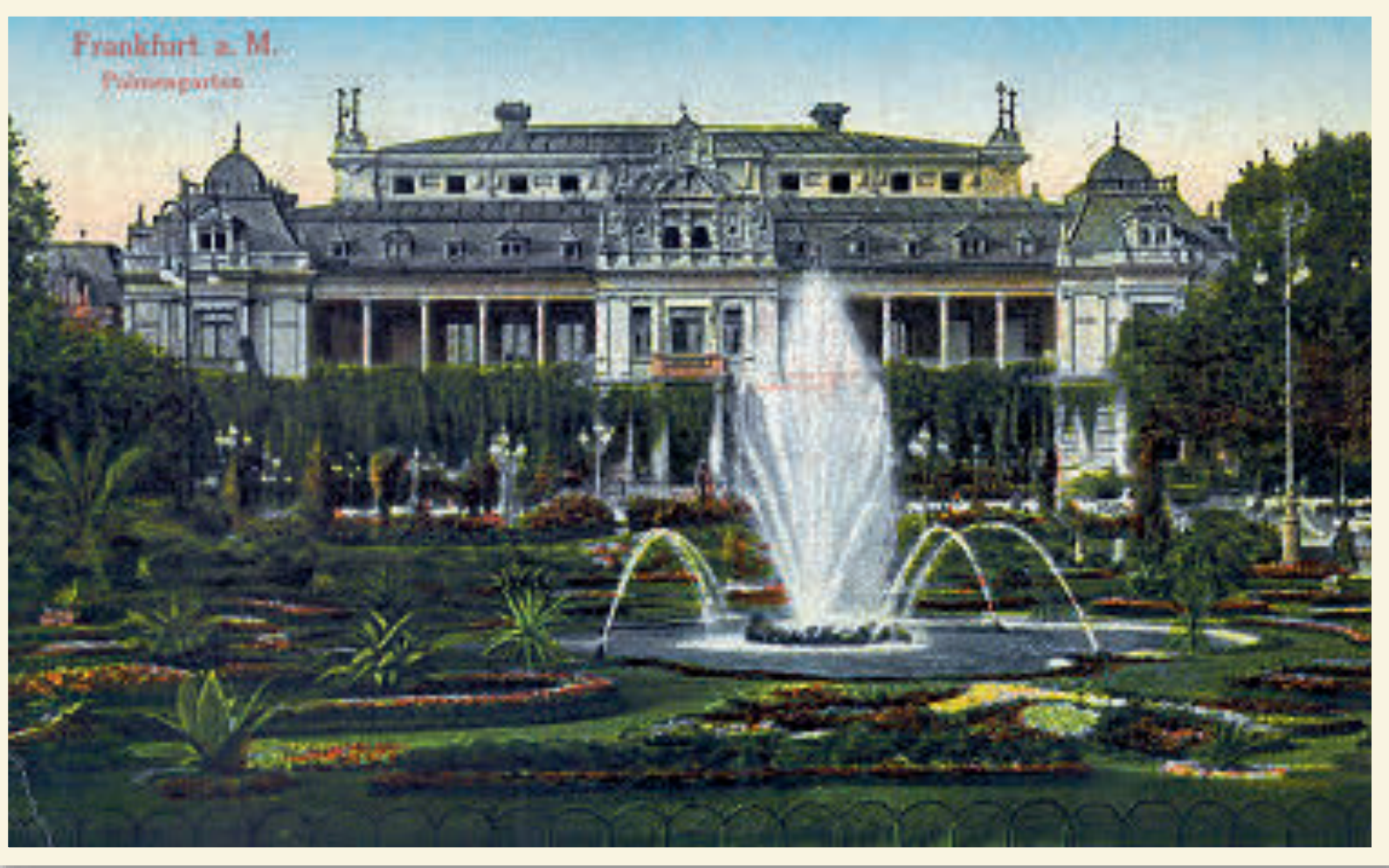




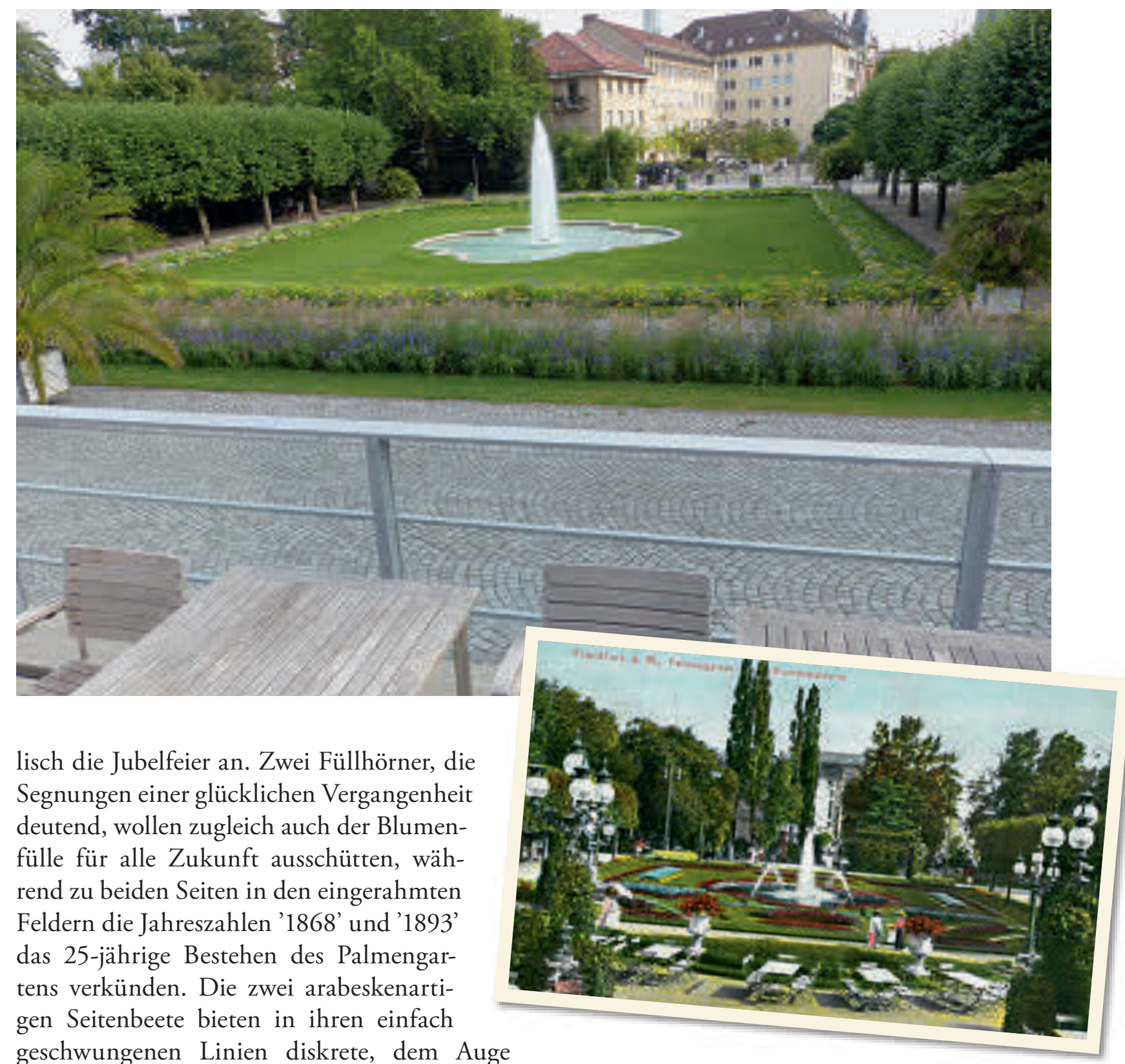
wohltuende Farbenkombinationen. Der grosse Springbrunnen mit seinen den Fröschen nachgebildeten Wasserspeiern lenkt unser Auge auf den in anmutigsten Farbenwechseln prangenden Blumenteppich, der als Paradestück des

Abb. 1 (Seite 134): Ansicht des Gesellschaftshauses mit Blumenparterre. Käufliche Ansichtskarte, abgeschickt am 23.5.1918.

Abb. 2 (oben): Ähnlicher Blick wie in Abb. 3, aufgenommen am 14.8.2015.

Abb. 2 (unten): Blick von der Terrasse des Gesellschaftshauses über das Blumenparterre in Richtung Palmengartenstraße. Käufliche Ansichtskarte, abgeschickt am 4.8.1912.

Palmengartens bekannt ist. In seinem Werke 'Rheinische Gärten' äussert sich Freiherr von OMPTEDA folgendermassen über unser Blumenparterre: 'Mag man auch die vegetabilische Teppichwirkerei, wie sie eine uralte Erbschaft aus den Gärten der arabischen Kalifen, der Renaissance und der Holländer des 17. Jahrhundes ist - und seit dem letzten Jahrzehnt unsere Rasenfläche vielfach überwucherte - als 'zopfigen Ungeschmack', als naturwidrige Nachahmung einer kunstvoll ausgelegten 'Schüssel italienischen Salates' grundsätzlich ablehnen: hier, wo einer grossen, schaulustigen Menge die hohe Schule modernster Kunstgärtnerei vorgeführt werden soll, erscheint die heitere, farbenpräch- 

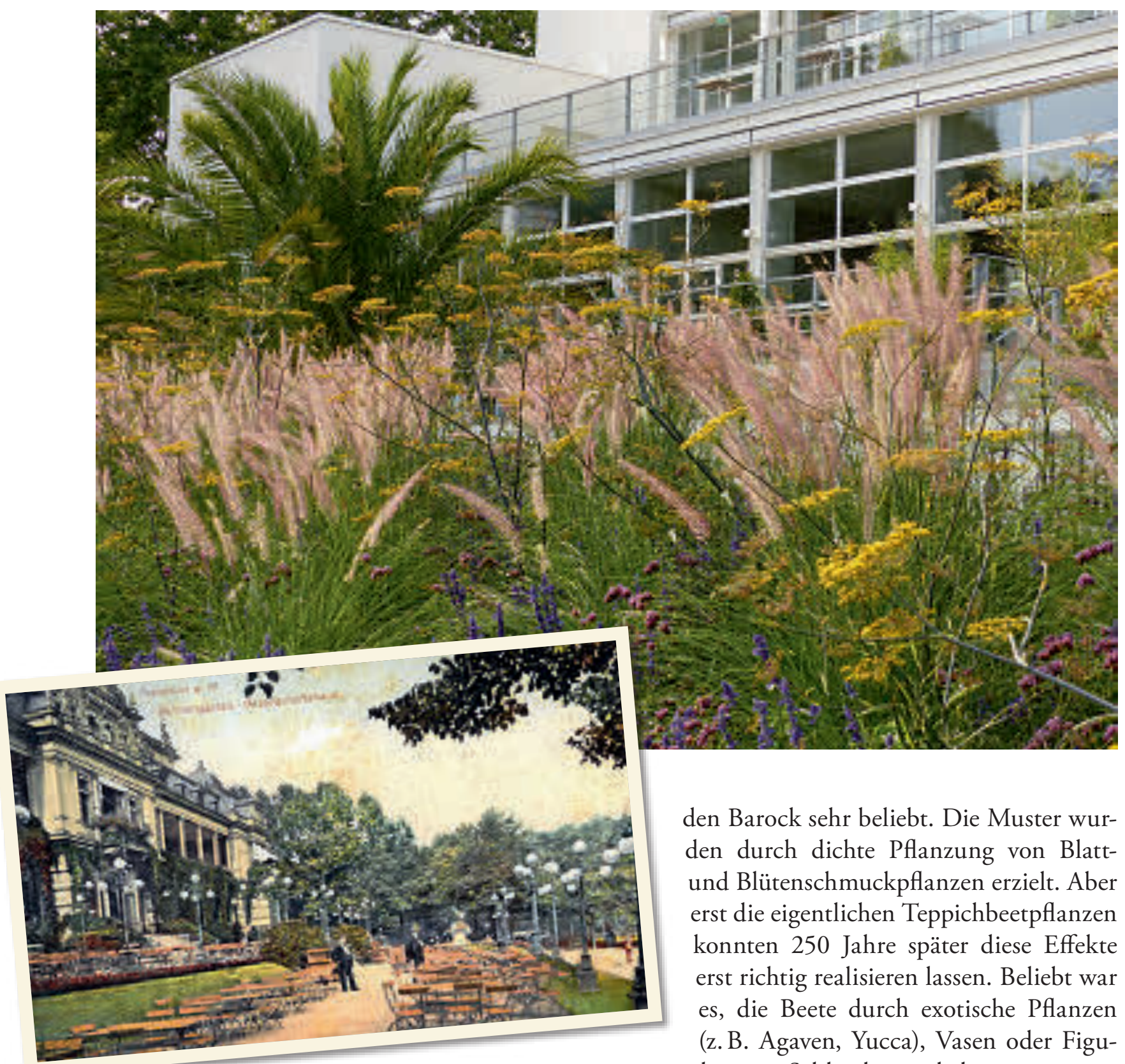

den Barock sehr beliebt. Die Muster wurden durch dichte Pflanzung von Blattund Blütenschmuckpflanzen erzielt. Aber erst die eigentlichen Teppichbeetpflanzen konnten 250 Jahre später diese Effekte erst richtig realisieren lassen. Beliebt war es, die Beete durch exotische Pflanzen (z. B. Agaven, Yucca), Vasen oder Figuren zu krönen. Schlossherren liebten es, von einer erhöhten Terrasse aus die Blumenteppiche

tige, stilisierte Entfaltung eines auserwählten Blumenflors durchaus an ihrem Platze. Wie ein festlicher Teppich rollt sich diese bunte Pracht aus dem reich aufgebauten Hauptportale hervor, die Terrassen hinab; wie ein voller, heiterer, stimmungsvoller Akkord bewillkommnet sie die erwartungsvollen Besucher und ladet sie gastlich ein, das für sie bereitete Haus zu betreten.'..."

\section{Lange Tradition der Teppichbeete}

Teppichbeete waren schon in der Renaissance (Kernzeit im 15. u. 16. Jh.) und im nachfolgen-
Abb. 4 (oben): Sommerflor auf der Böschung am Gesellschaftshaus, 100 Jahre später aufgenommen als Abb. 5 (14.8.2015).

Abb. 5 (unten): Böschung und Terrasse am Gesellschaftshaus. Käufliche Ansichtskarte, abgeschickt am 24.1.1915.

Abb. 6 (Seite 137 oben): Die neue sachliche Fassade des Gesellschaftshauses. Käufliche Ansichtskarte, Ende1920er-Jahre.

Abb. 7 (Seite 137 unten): Aktueller Zustand des Gesellschaftshauses mit Blumenparterre, aufgenommen am 14.8.2015. 
quasi als Fortsetzung der Teppiche im Schloss zu betrachten. Beete direkt vor der Gartenfront eines Schlosses, Palastes oder Herrenhauses werden als (Blumen)parterre bezeichnet.

\section{Heinrich Siesmayer und die Teppichbeete} Mit der Anlage der Teppichbeete im Palmengarten, aber auch in anderen Parks und Gärten, hat sich der Palmengarten-Gründer Heinrich Siesmayer (1817-1900) einen Namen gemacht. Später sprach man in diesem Zusammenhang sogar von Siesmayerei. Die Anlage von Teppichbeeten wurde Mitte des 19. Jh. modern, nachdem Pflanzenjäger viele hierfür geeignete Gewächse nach Europa gebracht hatten.

Die Bezeichnung Teppichbeet wurde erstmals in der Deutschen Gartenzeitung 1867 aufgegriffen, und nur zwei Jahre später gab es bereits TeppichbeetWettbewerbe in Namur und Hamburg. In der Deutschen Gartenzeitung, dem Organ der Vereinigten Gartenbau-Gesellschaften, sowie in
Möllers Deutscher Gärtner-Zeitung erschienen Ende des 19. Jh. und zu Beginn des 20. Jh. zahlreiche Beiträge zur Gestaltung von Teppichbeeten. Günstigen Einfluss auf die Entwicklung von Teppichbeeten hatte der Bau von Eisenbahnstrecken, weil dadurch Pflanzenraritäten leichter und schneller aus größerer Entfernung herbeigeschafft werden konnten. Bessere Gewächshaustechnik erlaubte es, empfindliche Pflanzen für die Teppichbeete besser vorzukultivieren bzw. zu überwintern. Auf erhöhten Beeten war es beliebt, exotische Pflanzen mit gro-

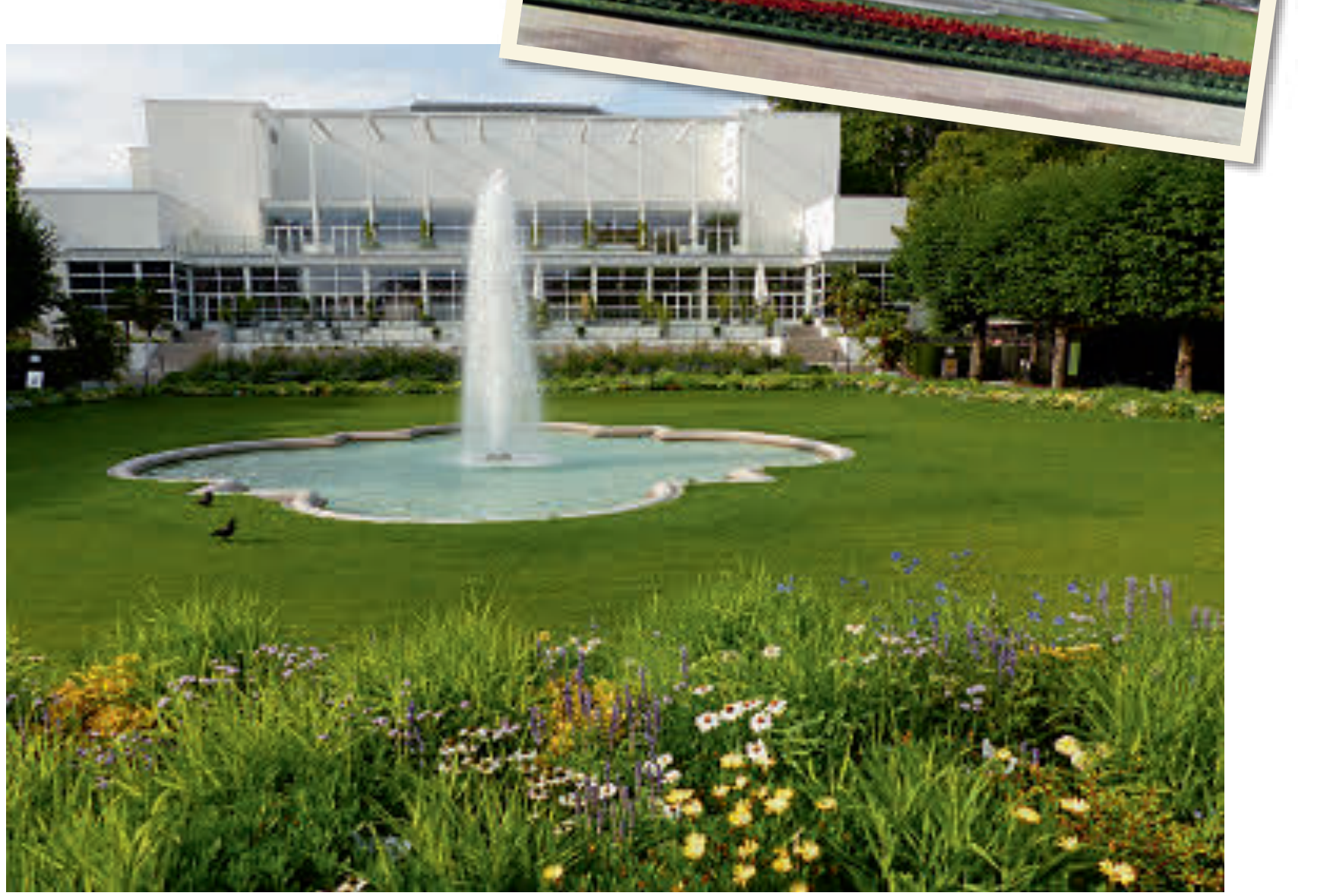




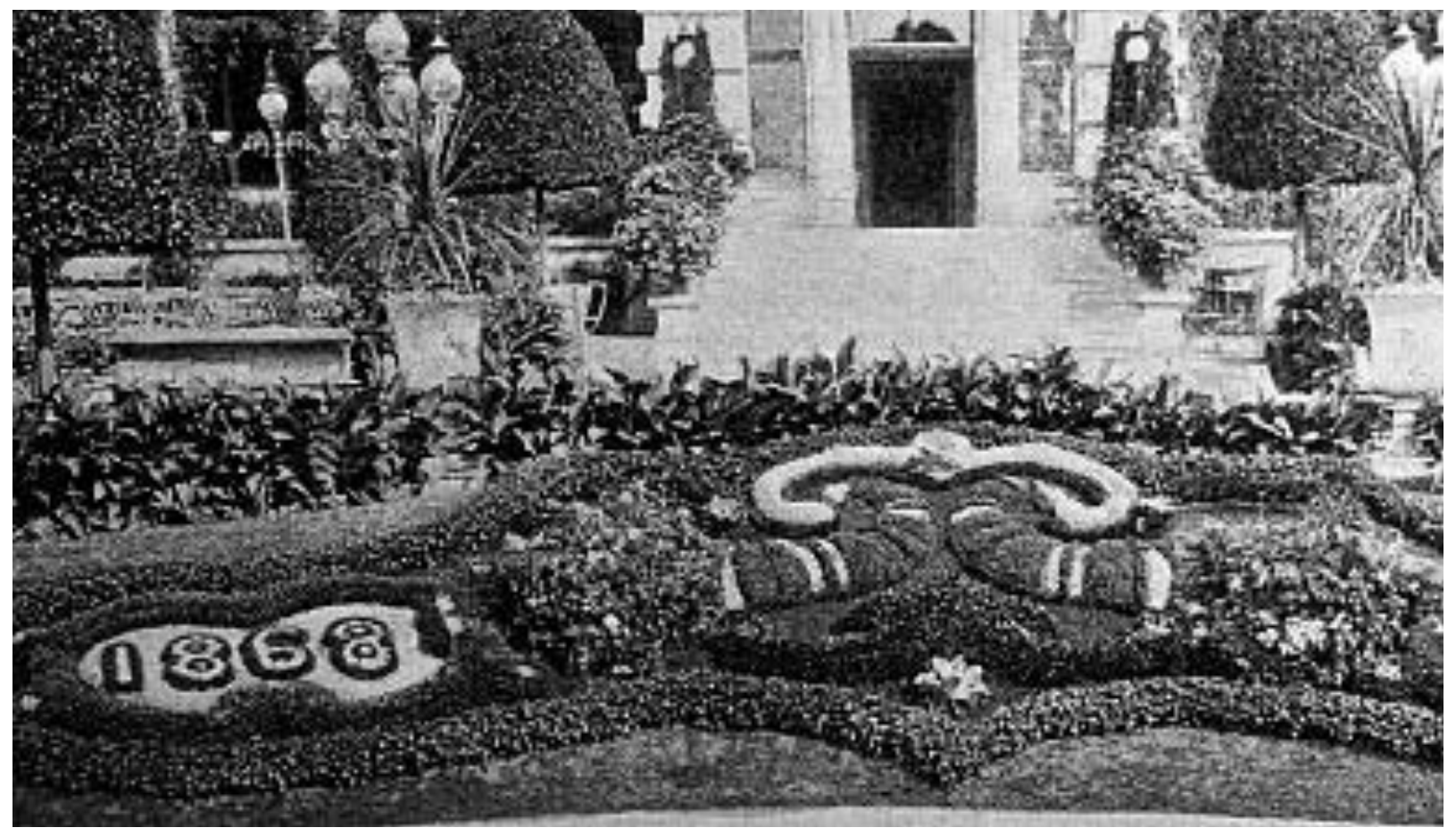

ßen Blättern zu integrieren wiez. B. Zwerg-Palme (Chamaerops humilis), Drachenbaum (Dracaena), Banane (Musa), Rizinus (Ricinus communis), Indisches Blumenrohr (Canna indica) oder andere tropische Blattschmuckstauden.

Flächig-dekorative Parterregärten waren oft aus mehreren Teil-Ornamenten zusammengesetzt. Muster und Pflanzenzusammensetzungen wurden jährlich gewechselt. Beliebt war es, Neuheiten zu verwenden wie z. B. die gerade erst im 19. Jh. eingeführten Begonien, Echeverien oder Zigarettenblümchen (Cuphea ignea). In Musterbüchern gab es viele Anregungen zur Gestaltung von Teppichbeeten. Stiefmütterchen in allen Farbabstufungen galten als sehr geeignet für Frühlingsbeete.

Der Frankfurter Palmengarten war ein Vorbild bei der Entwicklung neuster Teppichbeetpflanzungen. Auch Heinrich Siesmayers Sohn Philipp (1862-1935) war den Teppichbeeten sehr zugeneigt und setzte die "Siesmayerei" in alter Familientradition fort. Er ließ sich auch durch die floralen Muster des Jugendstils beeinflussen. Im Palmengarten ließ Siesmayers Nachfolger, August Siebert, die Tradition der Teppichbeete weiterleben und entwickelte sie auch weiter.

\section{Das Blumenparterre heute}

Mit der Neugestaltung der Fassade in den 1920er-Jahren im sachlichen Bauhaus-Stil wurden die Beete entsprechend sparsamer gestaltet und die Bepflanzung auf die Randbereiche beschränkt. Bis in heutige Zeit bewahrt der Palmengarten die Tradition, die Randbeete um die zentrale Rasenfläche mit dem Brunnen attraktiv zu bepflanzen. Jedes Jahr werden auf dem Parterre die Randbordüren mit neuen Mustern gepflanzt. Wie auch früher gibt es jedes Jahr im Frühlings- und Sommerflor neue Farb- und Pflanzenkombinationen. Aufgegriffen wird dabei auch immer wieder die Farbe Weiß, die mit der weißen Front des Gesellschaftshauses korrespondiert. Bezüge werden, wenn es sich ergibt, auch zum Thema der großen Jahresausstellung hergestellt. Als sich im Jahr 2014 im Palmengarten alles um Duft drehte, waren viele würzige Kräuter wie Salbei, Fenchel oder Basilikum in den Sommerflor integriert.

\section{Literatur}

Siebert, A. 1895: Der Palmengarten zu Frankfurt a. M. - Berlin.

Abb. 8: Ausschnitt aus der Jubiläumsbepflanzung des mittleren Teppichbeetes im Jahr 1893, Abbildung aus dem Gartenführer von 1895. 